\title{
Conservation Laws of Some Physical Models via Symbolic Package GeM
}

\author{
Rehana Naz, ${ }^{1}$ Imran Naeem, ${ }^{2}$ and M. Danish Khan ${ }^{2}$ \\ ${ }^{1}$ Centre for Mathematics and Statistical Sciences, Lahore School of Economics, Lahore 53200, Pakistan \\ ${ }^{2}$ Department of Mathematics, School of Science and Engineering, LUMS, Lahore Cantt 54792, Pakistan
}

Correspondence should be addressed to Rehana Naz; rehananaz_qau@yahoo.com

Received 10 May 2013; Accepted 13 June 2013

Academic Editor: Chaudry Masood Khalique

Copyright (C) 2013 Rehana Naz et al. This is an open access article distributed under the Creative Commons Attribution License, which permits unrestricted use, distribution, and reproduction in any medium, provided the original work is properly cited.

\begin{abstract}
We study the conservation laws of evolution equation, lubrication models, sinh-Poisson equation, Kaup-Kupershmidt equation, and modified Sawada-Kotera equation. The symbolic software GeM (Cheviakov (2007) and (2010)) is used to derive the multipliers and conservation law fluxes. Software GeM is Maple-based package, and it computes conservation laws by direct method and first homotopy and second homotopy formulas.
\end{abstract}

\section{Introduction}

The study of conservation laws plays a vital role in analysis, solution, and reductions of PDEs. For the PDEs, the conservation laws are used in wide variety of applications, for example, inverse scattering transform in soliton solutions [1], bi-Hamiltonian structures and recursion operators [2], Lax operators [3], and derivation of conserved quantities for jet flows [4].

Different methods have been developed so far for the construction of conservation laws and are well documented in [5-7]. In the last few decades, the researchers focused on the development of symbolic computational packages based on different approaches of conservation laws. These packages work with either Mathematica or Maple. The development of symbolic computational packages gives relief to perform complicated and tedious algebraic computation. Recently, several computational packages have been developed, for example, CONDENS.M by Göktaş and Hereman [8], RUDCE by Wolf et al. [9-11], TransPDEDensity.m by Adams and Hereman [12], GeM by Cheviakov [13, 14], Vessiot suite by Anderson and Cheb-Terrab [15], ConservationLawsMD.m by Poole and Hereman [16], and SADE by Rocha Filho and Figueiredo [17].

In this paper, we will use GeM package [13] to compute the conservation laws for partial differential equations
(PDEs) arising in applications. GeM package works with Maple to obtain the symmetries and conservation laws of differential equations. In symmetry analysis, it first computes the overdetermined system of determining equations and then simplifies the system by Rif package routines. After simplification, a Maple command in GeM generates all symmetry generators of differential equation. In conservation laws analysis, GeM computes an overdetermined system of determining equation of conservation law multipliers, and then this system is simplified by Rif package which is solved by using the built-in Maple function pdsolve to get multipliers. After computing multipliers, the conservation laws fluxes are derived by one of the following four methods: direct method [18, 19], first homotopy formula [20], second homotopy formula [19], and scaling symmetry formula [21]. All these four methods have some limitations in their use. The direct method written in GeM [13] is a Maple implementation based on Wolf [11] program in REDUCE. For simple partial differential equation (PDE) systems and multipliers, direct method is used to calculate fluxes. It is also used if arbitrary functions are involved. The conservation laws fluxes for complicated PDEs or multipliers, not involving arbitrary functions, are established by using first and second homotopy formulas. The scaling symmetry method is used to compute fluxes for the scaling-homogeneous PDEs or/and multipliers. For the complicated scaling-homogeneous PDEs 
and/or multipliers involving arbitrary functions, this is only a systematic method for computing fluxes.

The evolution equations are important and arise in many applications. We compute the conservation laws of various nonlinear evolution equations using GeM Maple routines. This includes a $(1+1)$-dimensional evolution equation [22], lubrication models [23], sinh-Poisson equation [24], KaupKupershmidt equation [25], and modified Sawada-Kotera equation [26]. At last, we summarize and discuss our results.

\section{Multipliers and Conservation Laws Using GeM Maple Routines}

2.1. Evolution Equation. As a first example, consider the following evolution equation [22]:

$$
u_{t t}+a u_{x x}+b u+c u^{3}=0
$$

where $u(t, x)$ and $a, b, c$ are constants. We will explain this example in detail along with GeM Maple routines given in $[13,14]$. The variables and partial differential equation (PDE) (1) are defined in GeM by the following Maple commands.

$$
\begin{aligned}
& \text { With }(\mathrm{GeM}) \text { : } \\
& \text { gem_decl_vars }(\text { indeps }=[\mathrm{t}, \mathrm{x}], \operatorname{deps}=[\mathrm{u}(\mathrm{t}, \mathrm{x})]) ; \\
& \text { gem_decl_eqs }([\operatorname{diff}(\mathrm{u}(\mathrm{t}, \mathrm{x}), \mathrm{t}, \mathrm{t})+\mathrm{a} * \operatorname{diff}(\mathrm{u}(\mathrm{t}, \mathrm{x}), \mathrm{x}, \mathrm{x})+\mathrm{b} * \\
& \left.\mathrm{u}(\mathrm{t}, \mathrm{x})+\mathrm{c} * \mathrm{u}^{3}(\mathrm{t}, \mathrm{x})=0\right], \\
& \text { solve_for }=[\operatorname{diff}(\mathrm{u}(\mathrm{t}, \mathrm{x}), \mathrm{t}, \mathrm{t})]) .
\end{aligned}
$$

The option solve_for is used in the flux-computation routine, and actually it defines a set of leading derivatives the given PDE systems can be solved for.

Consider multipliers of the form $\Lambda=\Lambda\left(t, x, u, u_{t}, u_{x}\right)$. In GeM, we use the Maple routines,

det_eqs:=gem_conslaw_det_eqs $([\mathrm{t}, \mathrm{x}, \mathrm{u}(\mathrm{t}, \mathrm{x}), \operatorname{diff}(\mathrm{u}(\mathrm{t}, \mathrm{x})$, $\mathrm{t}), \operatorname{diff}(\mathrm{u}(\mathrm{t}, \mathrm{x}), \mathrm{x})])$ :

CL_multipliers:=gem_conslaw_multipliers();

simplified_eqs:=DEtools[rifsimp](det_eqs,

CL_multipliers, mindim=1),

to obtain the set of determining equations for the multipliers expressed in the simplified form as

$$
\begin{gathered}
\Lambda_{x x}=0, \quad \Lambda_{u}=0, \quad \Lambda_{x u_{x}}=0, \quad \Lambda_{u_{x} u_{x}}=0, \\
\Lambda_{t}=-\frac{a u_{x} \Lambda_{x}}{u_{t}}, \\
\Lambda_{u_{t}}=\frac{\Lambda-u_{x} \Lambda_{u_{x}}}{u_{t}}, \quad \text { with } a \neq 0, b \neq 0, c \neq 0 .
\end{gathered}
$$

To solve the system (2), we use the Maple command multipliers_sol:=pdsolve(simplified_eqs[Solved]),

and it yields

$$
\Lambda\left(t, x, u, u_{t}, u_{x}\right)=\left(c_{3} x+c_{1}\right) u_{t}+\left(-c_{3} a t+c_{2}\right) u_{x},
$$

where $c_{1}, c_{2}, c_{3}$ are arbitrary constants. We obtain three linearly independent conservation laws, arising from the multipliers

$$
\Lambda^{(1)}=u_{t}, \quad \Lambda^{(2)}=u_{x}, \quad \Lambda^{(3)}=x u_{t}-a t u_{x} .
$$

Next step is the derivation of conservation laws associated with multipliers given in (4). The Maple command

$$
\text { gem_get_CL_fluxes(multipliers_sol) }
$$

computes the flux expressions by the direct method. For the multipliers (4), we have the following conservation laws fluxes:

$$
\begin{gathered}
\phi^{(1)}=\frac{1}{2} u_{t}^{2}-\frac{1}{2} a u_{x}^{2}+\frac{1}{4} c u^{4}+\frac{1}{2} b u^{2}, \quad \psi^{(1)}=a u_{x} u_{t}, \\
\phi^{(2)}=u_{t} u_{x}+b t u u_{x}+c t u^{3} u_{x}, \\
\psi^{(2)}=-\frac{1}{2} u_{t}^{2}-c t u^{3} u_{t}-b t u u_{t}+\frac{1}{2} a u_{x}^{2}, \\
\phi^{(3)}=-\frac{1}{2} a x u_{x}^{2}-a t u_{t} u_{x}+\frac{1}{2} x u_{t}^{2}+\frac{1}{4} c x u^{4}+\frac{1}{2} b x u^{2}, \\
\psi^{(3)}=-\frac{1}{4} a c t u^{4}-\frac{1}{2} a b t u^{2}+\frac{1}{2} a t u_{t}^{2}+a x u_{x} u_{t}-\frac{1}{2} a^{2} t u_{x}^{2} .
\end{gathered}
$$

The multipliers given in (4) do not involve arbitrary functions, so homotopy formulas can be used to compute fluxes. We call the routine for first homotopy method

$$
\begin{aligned}
& \text { gem_get_CL_fluxes(multipliers_sol, } \\
& \text { method="Homotopy1") }
\end{aligned}
$$

to get the following expressions for conservation law fluxes:

$$
\begin{gathered}
\phi^{(1)}=\frac{1}{4} c u^{4}+\frac{1}{2} u_{t}^{2}+\frac{1}{2} a u u_{x x}+\frac{1}{2} b u^{2}, \\
\psi^{(1)}=-\frac{1}{2} a u u_{t x}+\frac{1}{2} a u_{x} u_{t}, \\
\phi^{(2)}=-\frac{1}{2} u u_{t x}+\frac{1}{2} u_{t} u_{x}, \\
\psi^{(2)}=\frac{1}{4} c u^{4}+\frac{1}{2} a u_{x}^{2}+\frac{1}{2} u u_{t t}+\frac{1}{2} b u^{2}, \\
\phi^{(3)}=\frac{1}{4} c u^{4} x+\frac{1}{2} a u u_{x}+\frac{1}{2} a t u u_{t x}-\frac{1}{2} a t u_{t} u_{x} \\
+\frac{1}{2} x u_{t}^{2}+\frac{1}{2} a x u u_{x x}+\frac{1}{2} b x u^{2}, \\
\psi^{(3)}=-\frac{1}{4} a c t u^{4}-\frac{1}{2} a u u_{t}-\frac{1}{2} a x u u_{t x}-\frac{1}{2} a^{2} t u_{x}^{2} \\
+\frac{1}{2} a x u_{t} u_{x}-\frac{1}{2} a t u u_{t t}-\frac{1}{2} a b t u^{2} .
\end{gathered}
$$

For second homotopy formula, the Maple command

$$
\begin{aligned}
& \text { gem_get_CL_fluxes(multipliers_sol, } \\
& \text { method="Homotopy2") }
\end{aligned}
$$

yields divergence expressions in the same form as in (6). 
TABLE 1: Multipliers and conserved vectors for PDE (14).

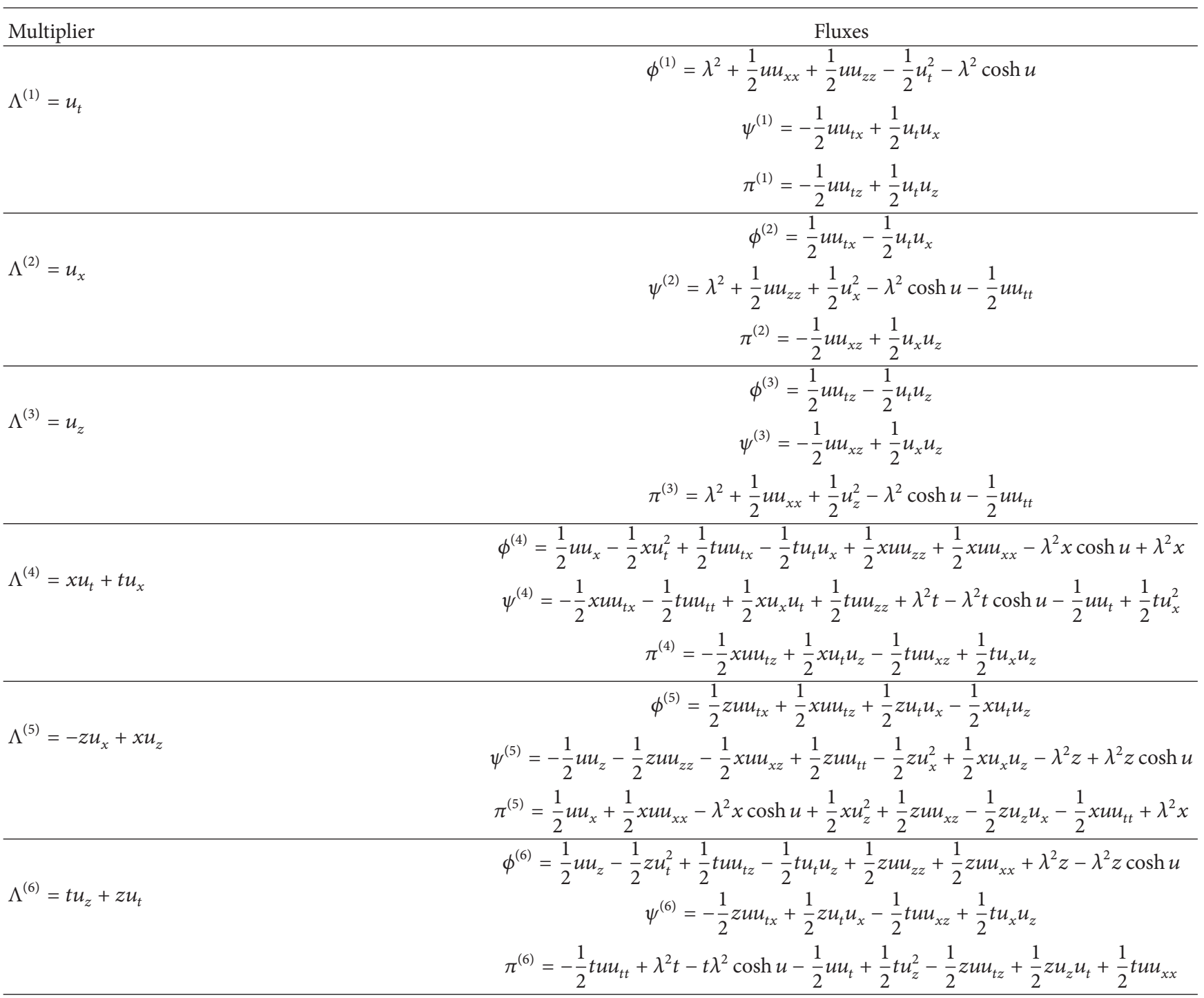

The PDE (1) has no scaling symmetry; therefore, we cannot apply the scaling symmetry formula here for derivation of fluxes.

2.2. Lubrication Models. Now we will study two lubrication models for conservation laws point of view. Gandarias and Medina [23] performed the symmetry analysis of lubrication model

$$
u_{t}=f(u) u_{x x x x}
$$

where $f$ is an arbitrary function. For $f(u)=c(u+b)^{a}$ and $f(u)=\gamma e^{\alpha u}$, this equation has some extra symmetry [23]. Without loss of generality, take $f(u)=u+b$ in (7); we have

$$
u_{t}=(u+b) u_{x x x x}
$$

where $b$ is arbitrary constant. Consider the multipliers of form $\Lambda(t, x, u)$ in GeM Maple routines, and then we obtain the following four multipliers:

$$
\begin{aligned}
\Lambda^{(1)}(t, x, u) & =\frac{1}{(u+b)}, & \Lambda^{(2)}(t, x, u) & =\frac{x}{(u+b)}, \\
\Lambda^{(3)}(t, x, u) & =\frac{x^{2}}{2(u+b)}, & \Lambda^{(4)}(t, x, u) & =\frac{x^{3}}{6(u+b)} .
\end{aligned}
$$

The fluxes associated with the multipliers given in (7) are computed by homotopy first method and are given by

$$
\begin{gathered}
\phi^{(1)}=\ln \left(\frac{u+b}{b}\right), \quad \psi^{(1)}=u_{x x x}, \\
\phi^{(2)}=x \ln \left(\frac{u+b}{b}\right), \quad \psi^{(2)}=-u_{x x}+x u_{x x x},
\end{gathered}
$$$$
\phi^{(3)}=\frac{1}{2} x^{2} \ln \left(\frac{u+b}{b}\right), \quad \psi^{(3)}=u_{x}-x u_{x x}+\frac{x^{2} u_{x x x}}{2},
$$ 
TABle 2: Multipliers and conserved vectors for PDE (15).

\begin{tabular}{|c|c|}
\hline Multiplier & Fluxes \\
\hline$\Lambda^{(1)}=1$ & $\begin{array}{c}\phi^{(1)}=u \\
\psi^{(1)}=-u_{x x x x}-\frac{5}{3} u^{3}-5 u u_{x x}-\frac{15}{4} u_{x}^{2}\end{array}$ \\
\hline$\Lambda^{(2)}=2 u^{2}+u_{x x}$ & $\begin{array}{c}\phi^{(2)}=\frac{2}{3} u^{3}+\frac{1}{2} u u_{x x} \\
\psi^{(2)}=4 u u_{x} u_{x x x}+\frac{1}{2} u_{t} u_{x}-4 u_{x}^{2} u_{x x}-\frac{9}{2} u u_{x x}^{2}+\frac{1}{2} u_{x x x}^{2}-2 u^{2} u_{x x x x}-u_{x x} u_{x x x x}-\frac{1}{2} u u_{t x}-10 u^{3} u_{x x}-2 u^{5}\end{array}$ \\
\hline$\Lambda^{(3)}=x+5 t u^{2}+\frac{5}{2} t u_{x x}$ & $\begin{array}{c}\phi^{(3)}=\frac{5}{3} t u^{3}+\frac{5}{4} u u_{x x}+x u \\
\psi^{(3)}=u_{x x x}-x u_{x x x x}+\frac{5}{4} t u_{x x x}^{2}-\frac{5}{4} t u u_{t x}-5 t u^{2} u_{x x x x}-\frac{5}{2} t u_{x x} u_{x x x x}+10 t u u_{x} u_{x x x}+\frac{5}{4} t u_{t} u_{x}-\frac{5}{3} x u^{3} \\
-5 t u^{5}-\frac{15}{4} x u_{x}^{4}-10 t u_{x}^{2} u_{x x}-\frac{45}{4} t u u_{x x}^{2}-5 x u u_{x x}-25 t u^{3} u_{x x}+\frac{15}{4} u u_{x}\end{array}$ \\
\hline$\Lambda^{(4)}=u u_{x x}+\frac{1}{2} u_{x}^{2}+\frac{1}{6} u_{x x x x}+\frac{4}{9} u^{3}$ & $\begin{array}{c}\phi^{(4)}=\frac{1}{9} u^{4}+\frac{1}{3} u^{2} u_{x x}+\frac{1}{6} u u_{x}^{2}+\frac{1}{12} u u_{x x x x} \\
\psi^{(4)}=-\frac{5}{6} u^{3} u_{x}^{2}+\frac{1}{12} u u_{x x x}^{2}-\frac{10}{27} u^{6}-\frac{11}{16} u_{x}^{4}-\frac{1}{12} u_{x x x x}^{2}-\frac{1}{12} u u_{t x x x}+\frac{1}{12} u_{x} u_{t x x}-\frac{1}{2} u_{x}^{2} u_{x x x x} \\
-\frac{1}{12} u_{t x} u_{x x}+u u_{x x} u_{x x x x}+\frac{1}{12} u_{t} u_{x x x}-\frac{1}{3} u^{2} u_{t x}-\frac{1}{12} u_{x} u_{x x} u_{x x x}+\frac{1}{3} u u_{t} u_{x}+\frac{1}{36} u_{x x}^{3} \\
-\frac{11}{4} u^{2} u_{x x}^{2}-\frac{20}{9} u^{4} u_{x x}-\frac{4}{9} u^{3} u_{x x x x}-\frac{7}{2} u u_{x}^{2} u_{x x}+\frac{1}{2} u^{2} u_{x} u_{x x x}\end{array}$ \\
\hline
\end{tabular}

$$
\begin{gathered}
\phi^{(4)}=\frac{1}{6} x^{3} \ln \left(\frac{u+b}{b}\right), \\
\psi^{(4)}=-u+x u_{x}-\frac{x^{2} u_{x x}}{2}+\frac{x^{3} u_{x x x}}{6} .
\end{gathered}
$$

We will get the same fluxes for (7) if we define higher order multipliers in GeM Maple routines.

Another interesting lubrication model is

$$
u_{t}+\frac{u_{x x x x}}{e^{u}}=0
$$

It is obtained by taking $f(u)=e^{-u}$ in (7). The GeM Maple routines yield the following four multipliers of form $\Lambda(t, x, u)$ :

$$
\begin{aligned}
\Lambda^{(1)}(t, x, u)=e^{u}, & \Lambda^{(2)}(t, x, u) & =x e^{u}, \\
\Lambda^{(3)}(t, x, u)=\frac{1}{2} x^{2} e^{u}, & \Lambda^{(4)}(t, x, u) & =\frac{1}{6} x^{3} e^{u} .
\end{aligned}
$$

The corresponding fluxes obtained by homotopy first method are

$$
\begin{gathered}
\phi^{(1)}=-1+e^{u}, \quad \psi^{(1)}=u_{x x x}, \\
\phi^{(2)}=x\left(-1+e^{u}\right), \quad \psi^{(2)}=-u_{x x}+x u_{x x x}, \\
\phi^{(3)}=\frac{1}{2} x^{2}\left(-1+e^{2}\right), \quad \psi^{(3)}=u_{x}-x u_{x x}+\frac{x^{2} u_{x x x}}{2}, \\
\phi^{(4)}=\frac{1}{6} x^{3}\left(-1+e^{u}\right), \\
\psi^{(4)}=-u+x u_{x}-\frac{x^{2} u_{x x}}{2}+\frac{x^{3} u_{x x x}}{6} .
\end{gathered}
$$

The conservation laws fluxes derived here can be used to find the solution of lubrication models and will be considered in future work.

2.3. sinh-Poisson Equation. The $(2+1)$-dimensional sinhPoisson equation is [24]

$$
u_{x x}+u_{z z}-u_{t t}=\lambda^{2} \sinh u
$$

where $u(t, x, z)$. The conservation laws for PDE (14) are derived here by using GeM routines. Consider the multipliers of form $\Lambda\left(t, x, u, u_{t}, u_{x}, u_{z}\right)$ in GeM routines, then it will yield six multipliers not containing any arbitrary function. The expression for fluxes is computed by using first homotopy formula. The multipliers and associated conserved vectors computed by first homotopy formula are given in Table 1.

2.4. Kaup-Kupershmidt Equation. Now, we will compute the conservation laws for the fifth order Kaup-Kupershmidt [25]:

$$
u_{t}=u_{x x x x x}+5 u u_{x x x}+\frac{25}{2} u_{x} u_{x x}+5 u^{2} u_{x}
$$

The GeM Maple routines yield three multipliers of the form $\Lambda\left(t, x, u, u_{t}, u_{x}, u_{x x}\right)$ for PDE (14). The first homotopy formula is applied to derive the expressions for conservation laws fluxes. One more multiplier can be computed if we consider higher order multipliers of the form $\Lambda\left(t, x, u, u_{t}, u_{x}, u_{x x}, u_{x x x}, u_{x x x x}\right)$. All the multipliers and associated conserved vectors for PDE (14) computed by first homotopy formula are presented in Table 2. 
TABLE 3: Multipliers and conserved vectors for PDE (16).

Multiplier

$\Lambda^{(1)}=1$

$\Lambda$

$\Lambda^{(2)}=u$

$\Lambda^{(3)}=u^{5}+u_{x x x x}-5 u_{x} u_{x x}$

$-5 u^{2} u_{x x}-5 u u_{x}^{2}$

$\Lambda^{(4)}=5 t u^{2} u_{x x}-25 t u^{2} u_{x x}+x u$

$-25 t u_{x} u_{x x}+5 t u_{x x x x}$
Fluxes

$\phi^{(1)}=u, \psi^{(1)}=5 u_{x} u_{x x}-u_{x x x x}+5 u^{2} u_{x x}+5 u u_{x}^{2}-u^{5}$

$\phi^{(2)}=\frac{1}{2} u^{2}$,

$\psi^{(2)}=5 u u_{x} u_{x x}-\frac{1}{2} u_{x x}^{2}+u_{x} u_{x x x}-\frac{5}{3} u_{x}^{3}+\frac{5}{2} u^{2} u_{x}^{2}+5 u^{3} u_{x x}-\frac{5}{6} u^{6}-u u_{x x x x}$

$\phi^{(3)}=\frac{1}{6} u^{6}-\frac{5}{4} u^{3} u_{x x}-\frac{5}{3} u u_{x} u_{x x}+\frac{1}{2} u u_{x x x x}$

$\psi^{(3)}=-\frac{1}{2} u_{x x x x}^{2}-\frac{1}{2} u^{10}-25 u u_{x}^{3} u_{x x}-\frac{25}{2} u_{x}^{2} u_{x x}^{2}-\frac{25}{2} u^{2} u_{x}^{4}+\frac{1}{2} u_{t} u_{x x x}$

$-\frac{5}{3} u_{t} u_{x}^{2}-\frac{5}{4} u^{2} u_{t} u_{x}-u^{5} u_{x x x x}+\frac{5}{3} u u_{x} u_{t x}-25 u^{3} u_{x}^{2} u_{x x}+5 u^{5} u_{x} u_{x x}$

$+5 u^{2} u_{x x} u_{x x x x}+5 u u_{x}^{2} u_{x x x x}-\frac{1}{2} u u_{t x x x}+\frac{5}{4} u^{3} u_{t x}+5 u^{7} u_{x x}+5 u^{6} u_{x}^{2}$

$+5 u_{x} u_{x x} u_{x x x x}-25 u^{2} u_{x} u_{x x}^{2}+\frac{1}{2} u_{x} u_{t x x}-\frac{1}{2} u_{t x} u_{x x}-\frac{25}{2} u^{4} u_{x x}^{2}$

$\phi^{(4)}=\frac{1}{6} t u^{6}-\frac{25}{4} t u^{2} u_{x}^{2}-\frac{25}{4} t u^{3} u_{x x}-\frac{25}{3} t u u_{x} u_{x x}+\frac{5}{2} t u u_{x x x x}+\frac{1}{2} x u^{2}$

$\psi^{(4)}=-\frac{25}{4} t u_{t} u_{x}+\frac{5}{4} u^{3} u_{x}-5 t u^{5} u_{x x x x}+\frac{1}{2} u_{x} u_{x x}+25 t u u_{x}^{2} u_{x x x x}+25 t u^{2} u_{x x} u_{x x x x}+5 x u u_{x} u_{x x}$

$-125 t u^{3} u_{x}^{2} u_{x x}-125 t u^{2} u_{x} u_{x x}^{2}-125 t u u_{x}^{3} u_{x x}-\frac{1}{2} x u_{x x}^{2}-\frac{5}{6} x u^{6}-\frac{5}{2} t u_{x x x x}^{2}-\frac{5}{3} x u_{x}^{3}+\frac{5}{3} u u_{x}^{2}$

$-\frac{3}{2} u u_{x x x}+25 t u^{5} u_{x} u_{x x}+25 t u_{x} u_{x x} u_{x x x x}-\frac{5}{2} t u^{10}-\frac{125}{2} t u_{x}^{2} u_{x x}^{2}-\frac{25}{3} t u_{t} u_{x}^{2}+\frac{5}{2} t u_{t} u_{x x x}$

$+\frac{5}{2} t u_{x} u_{t x x}+25 t u^{6} u_{x}^{2}+25 t u^{7} u_{x x}-\frac{5}{2} t u_{t x} u_{x x}-\frac{5}{2} t u u_{t x x x}+\frac{25}{4} t u^{3} u_{t x}$

$+x u_{x} u_{x x x}+5 x u^{3} u_{x x}-x u u_{x x x x}-\frac{125}{2} t u^{4} u_{x x}-\frac{125}{2} t u^{2} u_{x}^{4}+\frac{5}{2} x u^{2} u_{x}^{2}+\frac{25}{3} t u u_{x} u_{t x}$

$\phi^{(5)}=-\frac{1}{6} u^{8}+\frac{1}{2} u^{4} u_{x}^{2}+\frac{3}{2} u^{5} u_{x x}+\frac{14}{5} u^{3} u_{x} u_{x x}+\frac{28}{15} u^{2} u_{x}^{3}+\frac{5}{4} u_{x}^{4}$

$+\frac{27}{4} u u_{x}^{2} u_{x x}+\frac{7}{4} u^{2} u_{x} u_{x x x}-\frac{1}{4} u^{2} u_{x x}-\frac{1}{2} u^{3} u_{x x x x}+\frac{1}{3} u u_{x x} u_{x x x}$

$-\frac{2}{3} u u_{x} u_{x x x x}+\frac{5}{3} u_{x} u_{x x}^{2}+\frac{5}{3} u_{x}^{2} u_{x x x}+\frac{1}{2} u u_{t x}+\frac{1}{2} u_{t} u_{x}-\frac{1}{2} u_{x} u_{x x x x x}$

$\psi^{(5)}=\frac{2}{3} u u_{t x} u_{x x x}+\frac{1}{3} u_{x x x}^{3}+\frac{19}{18} u_{x}^{6}+\frac{1}{2} u_{x x}^{4}-8 u^{3} u_{x}^{2} u_{x x x x}-u_{x x} u_{x x x} u_{x x x x}$

$-9 u^{4} u_{x x} u_{x x x x}-7 u_{x}^{2} u_{x x} u_{x x x x}+\frac{140}{3} u^{3} u_{x}^{3} u_{x x}-\frac{20}{3} u^{7} u_{x} u_{x x}-\frac{28}{3} u u_{x}^{3} u_{x x x x}$

$+u u_{x x}^{2} u_{x x x x}+\frac{1}{4} u_{t} u_{x}^{3}+16 u u_{x}^{2} u_{x x} u_{x x x}+12 u^{3} u_{x} u_{x x} u_{x x x}+\frac{1}{2} u_{x} u_{t x x x x}-2 u u_{x} u_{x x x} u_{x x x x}$

$-14 u^{2} u_{x} u_{x x} u_{x x x x}-\frac{13}{4} u^{2} u_{x} u_{t x x}-\frac{1}{2} u^{2} u_{t} u_{x x x}+\frac{133}{6} u^{6} u_{x x}^{2}+10 u^{4} u_{x}^{4}-\frac{7}{3} u_{x}^{2} u_{t x x}$

$+u_{t} u_{x x}^{2}-\frac{1}{2} u_{x x} u_{t x x x}+\frac{1}{2} u_{t x x} u_{x x x}-\frac{1}{2} u_{t x} u_{x x x x}-u u_{x x} u_{t x x}+\frac{86}{3} u u_{x x} u_{x}^{4}+14 u^{2} u_{x}^{3} u_{x x x}$

$+3 u_{x} u_{x x}^{2} u_{x x x}-5 u u_{x} u_{x x}^{3}+4 u^{2} u_{x x}^{2} u_{x x x}+45 u^{4} u_{x} u_{x x}^{2}-3 u^{2} u_{x} u_{x x x}+10 u^{4} u_{x}^{2} u_{x x x}$

$+\frac{67}{2} u^{2} u_{x}^{2} u_{x x}^{2}-\frac{3}{2} u^{5} u_{t x}+\frac{3}{2} u^{4} u_{t} u_{x}-\frac{1}{4} u u_{x}^{2} u_{t x}+\frac{4}{3} u^{7} u_{x x x x}-\frac{20}{3} u^{9} u_{x x}$

$+\frac{15}{4} u^{2} u_{t x} u_{x x}-\frac{35}{6} u^{8} u_{x}^{2}-\frac{1}{2} u u_{t t}+\frac{1}{2} u^{3} u_{t x x x}+\frac{5}{9} u^{1} 2-\frac{1}{2} u u_{t} u_{x} u_{x x}+26 u^{5} u_{x}^{2} u_{x x}$

$+\frac{2}{3} u^{6} u_{x} u_{x x x}+u_{x} u_{x x x x}^{2}+u^{2} u_{x x x x}^{2}-\frac{17}{3} u^{3} u_{x x}^{3}+\frac{70}{3} u^{2} u_{x}^{5}-\frac{70}{9} u^{6} u_{x}^{3}-\frac{1}{2} u_{x}^{2} u_{x x x}^{2}$

$-\frac{1}{2} u^{4} u_{x x x}^{2}+\frac{40}{3} u_{x}^{3} u_{x x}^{2}-\frac{2}{3} u_{x}^{4} u_{x x x}+\frac{7}{3} u_{x} u_{t x} u_{x x}$

$+\frac{14}{5} u_{t} u^{2} u_{x}^{2}-\frac{4}{3} u_{t} u_{x} u_{x x x}+\frac{2}{3} u u_{x} u_{t x x x}-\frac{14}{5} u^{3} u_{x} u_{t x}$ 
2.5. Modified Sawada-Kotera Equation. Consider the fifth order modified SK equation:

$$
u_{t}=u_{x x x x x}-\left(5 u_{x} u_{x x}+5 u u_{x}^{2}+5 u^{2} u_{x x}-u^{5}\right)_{x} .
$$

For PDE (16), two conserved densities were derived by first computing Lax pair (see [26]). The higher order conservation laws fluxes exist for higher order multipliers and are not reported in [26]. Consider the multipliers of form $\Lambda\left(t, x, u, u_{t}, u_{x}, u_{t t}, u_{t x}, u_{x x}, u_{x x x}, u_{x x x x}\right)$ in GeM routines, then it will yield two simple and three higher order multipliers not containing any arbitrary function. The simple multipliers yield same fluxes as derived in [26], and three new fluxes corresponding to higher order multipliers are computed. The multipliers and associated conserved vectors computed by first homotopy formula are listed in Table 3 .

\section{Conclusions}

The conservation laws for the evolution equation, Benjamin equation, lubrication models, sinh-Poisson equation, KaupKupershmidt equation, and modified Sawada-Kotera equation were derived by using the symbolic software GeM. First of all, we considered the evolution equation, and the commands for all GeM Maple routines, were explicitly given. The first order multipliers were defined in GeM Maple routines and three multipliers were obtained. The expressions for fluxes were computed by direct method and first and second homotopy formulas and equivalent expressions for fluxes were obtained. The scaling symmetry method was not applicable here as no scaling symmetry exists for the nonlinear evolution equation. The conservation laws fluxes for the lubrication models, sinh-Poisson equation, KaupKupershmidt equation, and modified Sawada-Kotera equation were derived by the first homotopy formula. For the modified Sawada-Kotera equation, three new fluxes were derived.

The fluxes derived here can be used in constructing the solutions of underlying PDEs and will be considered in the future work.

\section{Conflict of Interests}

The authors declare that there is no conflict of interests.

\section{References}

[1] M. J. Ablowitz and P. A. Clarkson, Solitons, Nonlinear Evolution Equations and Inverse Scattering, vol. 149 of London Mathematical Society Lecture Note Series, Cambridge University Press, Cambridge, 1991.

[2] D. E. Baldwin and W. Hereman, "A symbolic algorithm for computing recursion operators of nonlinear partial differential equations," International Journal of Computer Mathematics, vol. 87, no. 5, pp. 1094-1119, 2010.

[3] V. Drinfel'd and V. Sokolov, "Lie algebras and equations of Korteweg-de vries type," Journal of Soviet Mathematics, vol. 30, no. 2, pp. 1975-2036, 1985.

[4] R. Naz, D. P. Mason, and F. M. Mahomed, "Conservation laws and conserved quantities for laminar two-dimensional and radial jets," Nonlinear Analysis, vol. 10, no. 5, pp. 2641-2651, 2009.

[5] G. W. Bluman, A. F. Cheviakov, and S. C. Anco, Applications of Symmetry Methods to Partial Differential Equations, vol. 168 of Applied Mathematical Sciences, Springer, New York, NY, USA, 1st edition, 2010.

[6] R. Naz, Symmetry solutions and conservation laws for some partial differ-ential equations in fluid mechanics [Ph.D. dissertation], University of the Witwatersrand, Johannesburg, South Africa, 2008.

[7] R. Naz, F. M. Mahomed, and D. P. Mason, "Comparison of different approaches to conservation laws for some partial differential equations in fluid mechanics," Applied Mathematics and Computation, vol. 205, no. 1, pp. 212-230, 2008.

[8] Ü. Göktaş and W. Hereman, "Symbolic computation of conserved densities for systems of nonlinear evolution equations," Journal of Symbolic Computation, vol. 24, no. 5, pp. 591-621, 1997.

[9] T. Wolf, A. Brand, and M. Mohammadzadeh, "Computer algebra algorithms and routines for the computation of conservation laws and fixing of gauge in differential expressions," Journal of Symbolic Computation, vol. 27, no. 2, pp. 221-238, 1999.

[10] T. Wolf, "A comparison of four approaches to the calculation of conservation laws," European Journal of Applied Mathematics, vol. 13, no. 2, pp. 129-152, 2002.

[11] T. Wolf, "Crack, LiePDE, ApplySym and ConLaw. section 4.3.5 and computer programon CD-ROM," in Computer Algebra Handbook, J. Grabmeier, E. Kaltofen, and V. Weispfenning, Eds., pp. 465-468, Springer, Berlin, Germany, 2002.

[12] P. J. Adams and W. Hereman, TransPDEDensityFlux.m: Symbolic computation of conserved densities and fluxes for systems of partial differential equations with transcendental nonlinearities, Scientific Software, 2002, http://inside.mines.edu/.

[13] A. F. Cheviakov, "GeM software package for computation of symmetries and conservation laws of differential equations," Computer Physics Communications, vol. 176, no. 1, pp. 48-61, 2007.

[14] A. F. Cheviakov, "Computation of fluxes of conservation laws," Journal of Engineering Mathematics, vol. 66, no. 1-3, pp. 153-173, 2010.

[15] I. M. Anderson and E. S. Cheb-Terrab, Differential geometry package, Maple Online Help, 2009, http://www.maplesoft .com/support/help/view.aspx?sid=26040.

[16] L. D. Poole and W. Hereman, "ConservationLawsMD.m: a mathematica package for the symbolic computation of conservation laws of polynomial systems of nonlinear PDEs in multiple space dimensions," scientific software, 2009, http://inside .mines.edu/ whereman/.

[17] T. M. Rocha Filho and A. Figueiredo, "[SADE] a Maple package for the symmetry analysis of differential equations," Computer Physics Communications, vol. 182, no. 2, pp. 467-476, 2011.

[18] S. C. Anco and G. Bluman, "Direct construction of conservation laws from field equations," Physical Review Letters, vol. 78, no. 15, pp. 2869-2873, 1997.

[19] S. C. Anco and G. Bluman, "Direct construction method for conservation laws of partial differential equations. II: general treatment," European Journal of Applied Mathematics, vol. 13, no. 5, pp. 567-585, 2002.

[20] W. Hereman, M. Colagrosso, R. Sayers et al., "Continuous and discrete homotopy operators and the computation of conservation laws," in Differential Equations with Symbolic Computation, D. Wang and Z. Zheng, Eds., pp. 249-285, Birkhäuser, 2005. 
[21] S. C. Anco, "Conservation laws of scaling-invariant field equations," Journal of Physics, vol. 36, no. 32, pp. 8623-8638, 2003.

[22] C. Bai, "Exact solutions for nonlinear partial differential equation: a new approach," Physics Letters A, vol. 288, no. 3-4, pp. 191-195, 2001.

[23] M. L. Gandarias and E. Medina, "Analysis of a lubrication model through symmetry reductions," Europhysics Letters, vol. 55, no. 2, pp. 143-149, 2001.

[24] A. H. Khater, W. Malfliet, D. K. Callebaut, and E. S. Kamel, "Travelling wave solutions of some classes of nonlinear evolution equations in $(1+1)$ and $(2+1)$ dimensions," Journal of Computational and Applied Mathematics, vol. 140, no. 1-2, pp. 469-477, 2002.

[25] V. P. Gomes Neto, "Fifth-order evolution equations describing pseudospherical surfaces," Journal of Differential Equations, vol. 249, no. 11, pp. 2822-2865, 2010.

[26] H. Guoliang and G. Xian-Guo, "An extension of the modified Sawada-Kotera equation and conservation laws," Chinese Physics B, vol. 21, no. 7, Article ID 070205, 2012. 


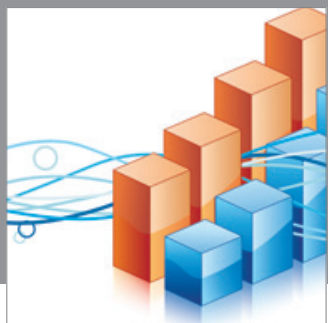

Advances in

Operations Research

mansans

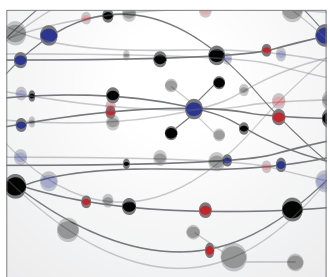

The Scientific World Journal
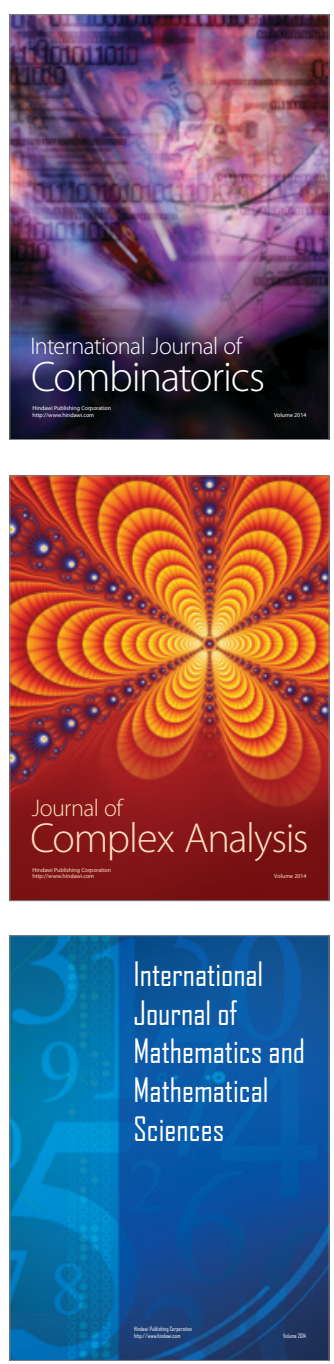
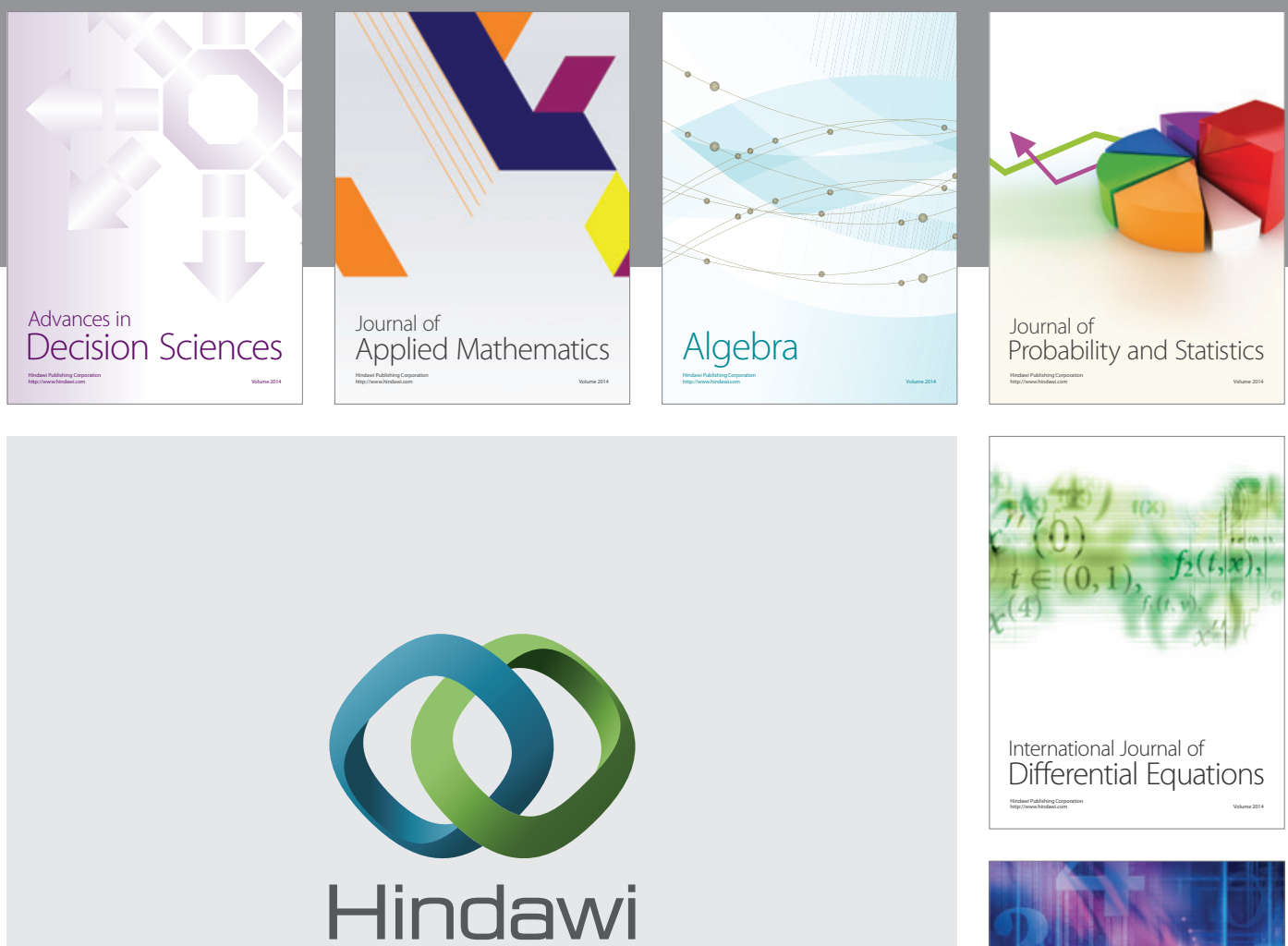

Submit your manuscripts at http://www.hindawi.com
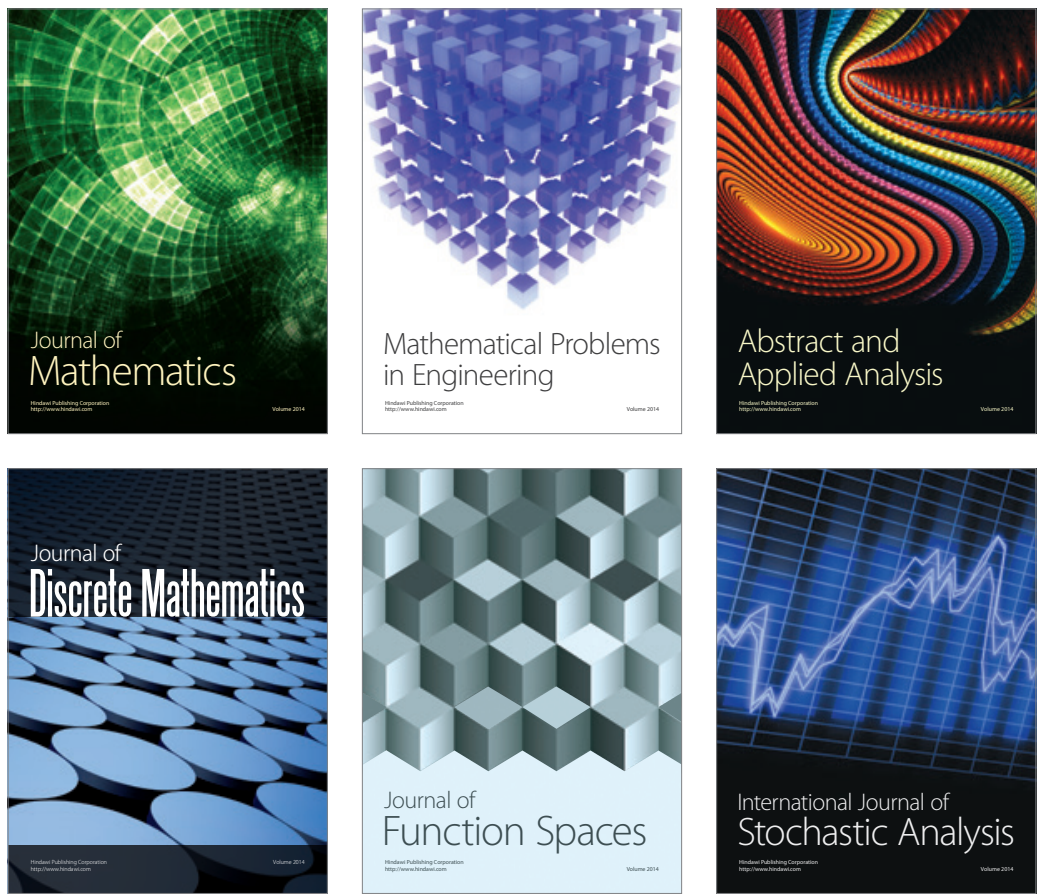

Journal of

Function Spaces

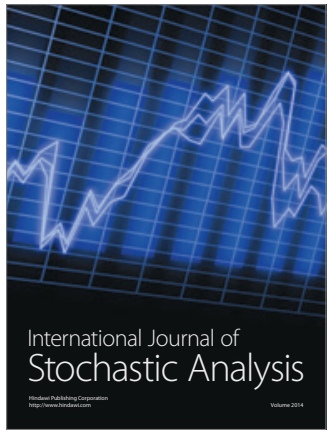

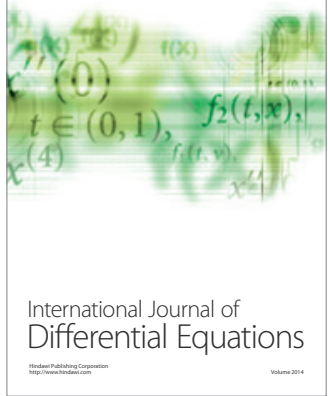
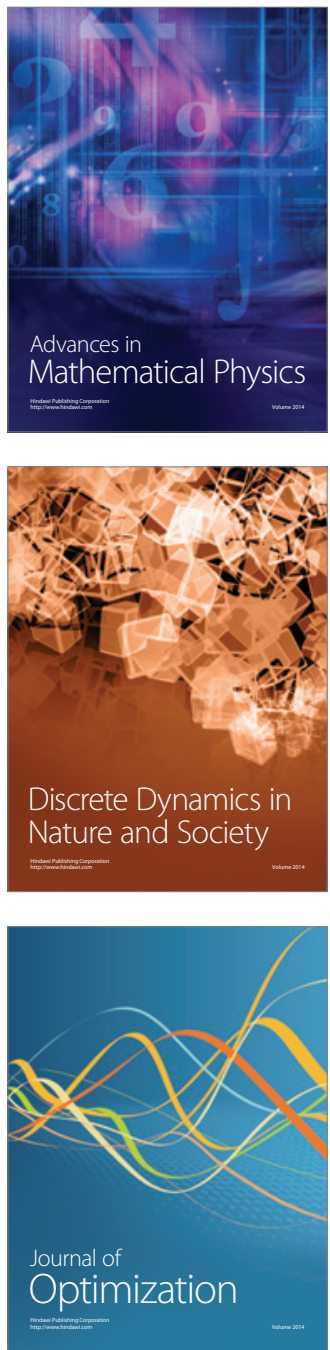